DOI: 10.26730/1999-4125-2020-4-36-41

УДК 621.9.048.6

\author{
ТЕХНОЛОГИЧЕСКИЕ ВОЗМОЖНОСТИ ВИБРООТДЕЛКИ ДЕТАЛЕЙ \\ ГРАНУЛИРОВАННЫМИ ОРГАНИЧЕСКИМИ СРЕДАМИ ИЗ ПРИРОДНЫХ \\ МАТЕРИАЛОВ
}

\title{
TECHNOLOGICAL POSSIBILITIES OF VIBRO-FINISHING OF PARTS WITH GRANULATED ORGANIC MEDIUM FROM NATURAL MATERIALS
}

\author{
Лебедев Валерий Александрович ${ }^{1}$, \\ канд. техн. наук, професcop, e-mail: va.lebidev@yandex.ru \\ Valery A. Lebedev ${ }^{1}$, C. Sc. in Engineering, Prof., \\ Крупеня Евгений Юрьевич ${ }^{2}$, \\ канд. техн. наук, доцент, e-mail: e.yu.kru@mail.ru \\ Evgeny Yu. Krupenya ${ }^{2}$, Candidate of Tech. Sciences, Associate Professor, \\ Шишкина Антонина Павловна ${ }^{2}$, \\ ст. преподаватель, e-mail: shishkina56646@ yandex.ru
} Antonina P. Shishkina ${ }^{2}$, senior lecturer

\footnotetext{
${ }^{1}$ Донской государственный технический университет, 344002, Россия, г. Ростов-на-Дону, пл. Гагарина, 1

${ }^{1}$ Don State Technical University, 1 Gagarin square, Rostov-on-don, 344002, Russia Federation ${ }^{2}$ Технологический институт (филиал) Донского государственного технического университета в г. Азове, 346780 Россия, Ростовская обл., г. Азов, ул. Промышленная, 1

${ }^{2}$ Technological Institute (branch) of the Don State Technical University in the city of Azov, 346780 Russia, Rostov region, Azov st. Industrial, 1

\begin{abstract}
Аннотация:
Показана сущность и область применения гранулированных органических сред из природных
\end{abstract} материалов, специфика этой группы обрабатывающих материалов в технологии изготовления деталей машин с учетом присущих им общих закономерностей протекания процесса в условиях виброобработки. Установлен механизм прочесса виброотделки косточковыми органическими средами и эффективные условия, обеспечивающие его реализачию, разработаны методы расчета показателей качества поверхности. Представлены модель процесса виброотделки поверхности деталей гранулами косточковых органических сред и результаты ее экспериментальной проверки; особенности сушки и вибропротирки деталей органическими средами.
}

Ключевье слова: виброобработка, гранулированная органическая среда, отделка поверхности, сушка, вибропротирка.

\section{Abstract:}

The essence and scope of application of granulated organic media from natural materials, the specificity of this group of processing materials in the technology of manufacturing machine parts, taking into account their inherent general laws of the process under vibration treatment are shown. The mechanism of the process of vibratory finishing with stone organic media and the effective conditions for its implementation have been established, methods for calculating surface quality indicators have been developed. A model of the process of vibrating the surface of parts with granules of stone fruit organic media and the results of its experimental verification are presented; peculiarities of drying and vibration cleaning of parts with organic media.

Key words: vibration finishing, granulated organic medium, surface finish, drying, vibration wiping. 
Гранулированные органические среды из природных материалов представляют собой самостоятельную группу обрабатывающих сред, состоящих из гранул косточек фруктовых деревьев, элементов из древесины, гранул, полученных в результате дробления стержней початковых растений [1]. Особенность этих обрабатывающих сред обусловлена их специфическими биолого-химическими свойствами, сформированными у них на этапах созревания переработки и хранения. Благодаря именно этим биолого-химическим свойствам возрастает роль органических обрабатывающих сред на операциях финишной отделки и полировки поверхностей сложнопрофильных деталей, высокоточных деталей гидроаппаратуры, электротехнических изделий, медицинских инструментов, бытовых и ювелирных изделий, для придания им эстетического и товарного вида; при подготовке поверхностей для нанесения функциональных покрытий. Органические среды обогащают ассортимент современной виброобработки, расширяют возможности обработки металлов и сплавов различной твердости - от алюминия до закаленной стали и чугуна и от нержавеющей стали до золота и платины, обеспечивают проведение сухой обработки, не предусматривающей организацию систем отвода сточных вод. В качестве базового технологического оборудования для реализации виброотделки деталей органическими средами наиболее рационально использовать вибростанки с кольцевой рабочей камерой.

Моделирование процесса виброотделки деталей косточковыми органическими средами.

Считая органическую гранулированную среду сплошной, используя гидродинамическую аналогию и пренебрегая тепловыми эффектами в гранулированной среде, выражение плотности потока энергии можно представить в виде:

$$
\mathrm{E}=\left(\frac{\rho_{\mathrm{oc}} \cdot \mathrm{V}^{2}}{2}+\mathrm{p}\right) \cdot V, \kappa \Gamma / \mathrm{c}^{3}
$$

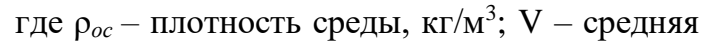
скорость потока среды, м/с; $p$ - внешнее и внутреннее «гидростатическое давление» в среде, Па;

При фиксированном направлении потока относительно поверхности предложено выражение для скорости металлосъема, м/с:

$$
\gamma_{\mathrm{M}}=\frac{E}{\Sigma},
$$

где ${ }^{\Sigma}$ - модуль сопротивления разрушению поверхности, Па.

Соотношение (2) качественно описывает установленную закономерность, состоящую в том, что повышение скорости и «давления» рабочей среды независимо увеличивает скорость металлосъема с поверхности.

В результате анализа теоретических и экспериментальных исследований движения гранулированных сред в рабочих камерах вибромашин установлено, что технологический эффект обработки косточковыми органическими средами будет достигнут при соблюдении следующих условий:

- образование в рабочей камере стационарного циркуляционного движения среды по слабо эксцентричным (близким к окружности) эллипсам, обеспечиваемого выполнением критериев:

$$
\frac{H}{L} \cong 1 ; \frac{H}{d_{г р}}>6 ; \frac{(A 2 \pi f)^{2}}{g H}<0,5,
$$

где $H$ - высота загрузки рабочей камеры, мм; $L-$ ширина сечения камеры, мм; $d_{\text {гр }}-$ средний

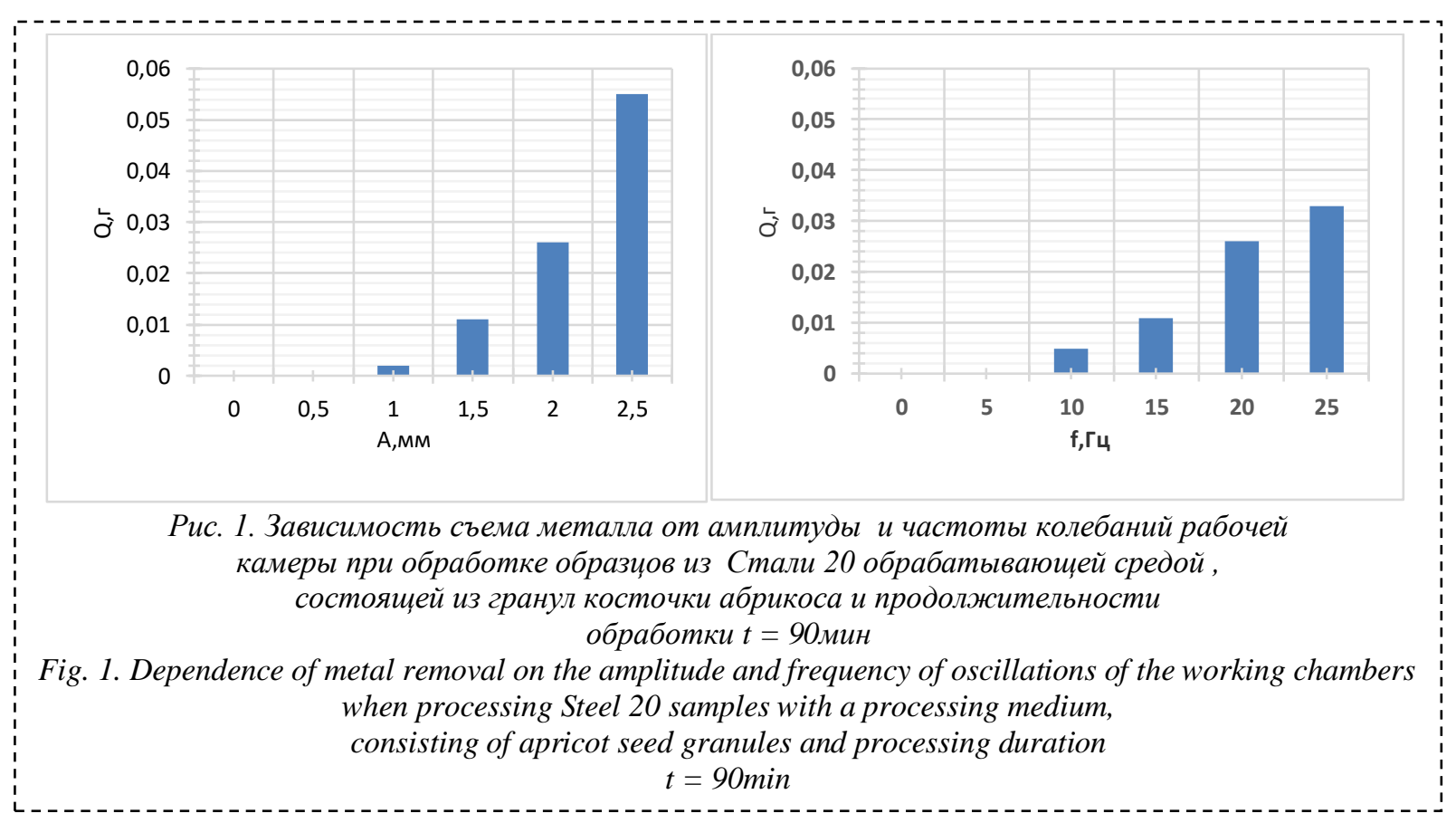


Таблица 1 . Величины модуля сопротивления разрушению $\Sigma$, Па

Table 1. Values of the modulus of resistance to destruction $\Sigma, \mathrm{Pa}$

\begin{tabular}{|c|c|c|c|}
\hline \multirow{2}{*}{ Материал } & \multicolumn{3}{|c|}{ Косточковая органическая среда } \\
\cline { 2 - 4 } & Opex & Вишня & Абрикос \\
\hline В95 & $8,27 \times 10^{10}$ & $8,35 \times 10^{10}$ & $8,31 \times 10^{10}$ \\
\hline Д16 & $7,145 \times 10^{10}$ & $7,219 \times 10^{10}$ & $7,178 \times 10^{10}$ \\
\hline Сталь 20 & $14,4 \times 10^{10}$ & $14,53 \times 10^{10}$ & $14,5 \times 10^{10}$ \\
\hline
\end{tabular}

Таблица 2. Результаты сравнительного анализа экспериментальных и расчетных значений величины скорости металлосъема

Table 2. Results of a comparative analysis of experimental and calculated values of the rate of metal removal

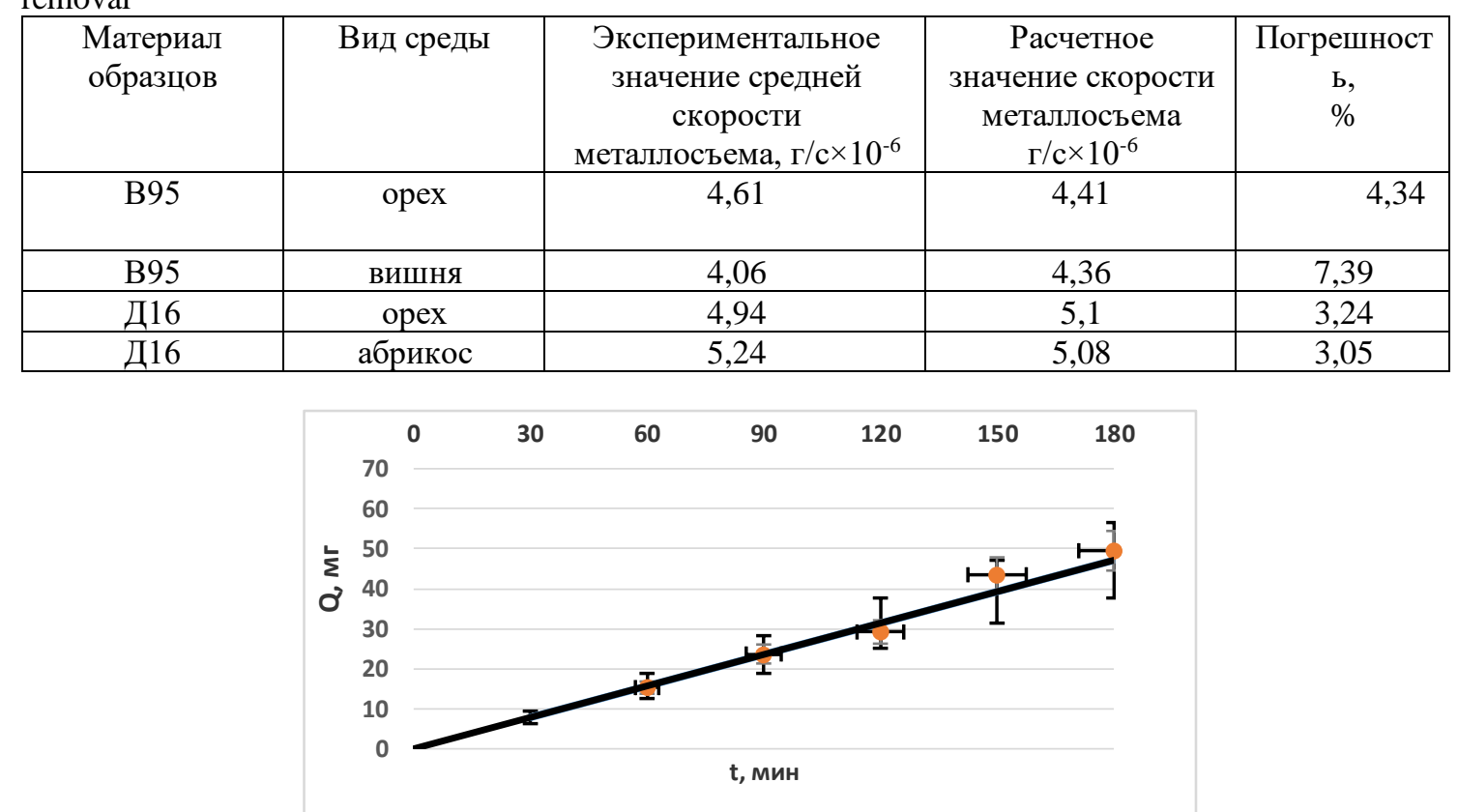

Рис. 2. Изменение съема в зависимости от продолжительности обработки при $A=2,5 м м, f=35 Г и$; материал образиов - сплав B95, обрабатывающая среда - гранульь вишни

Fig. 2. Change in removal depending on the duration of processing at $A=2.5 \mathrm{~mm}, f=35 \mathrm{~Hz}$; sample material - alloy B95, processing medium - cherry granules

размер гранулы среды, мм;

- амплитуда $A$ и частота $f$ колебаний рабочей камеры превышают пороговые значения $A_{0}=$ $1 \mathrm{~mm}, f_{0}=10-15$ Цц, ниже которых, как показали результаты экспериментальных исследований (рис. 1), эффект воздействия обрабатывающей среды на поверхность обрабатываемой детали, обуславливающий съем с нее материала, отсутствует.

Кроме того, установлено, что величина контактного давления потока среды на обрабатываемую поверхность зависит от высоты загрузки обрабатывающей среды в рабочую камеру, а скорость потока в состоянии установившегося движения потока по вибрирующей поверхности пропорциональна виброскорости самой поверхности.

Выразив высоту загрузки через ширину сечения рабочей камеры согласно (3)

$$
\begin{gathered}
p=\rho_{\text {ос }} g H=\underset{\text { ос }}{\rho_{0}} g L \\
\text { максимальную }
\end{gathered} \underset{\text { скорость }}{\text { циркуляционнового }}
$$

движения среды внутри камеры соотношением

$$
V=K_{\text {д }} A \pi f,
$$

где $K_{\text {д }}-$ коэффициент диссипации, выражение для плотности потока энергии (1) преобразовано к виду:

$$
E=K_{\text {д }} \pi g \rho_{\text {ос }} L\left(\mathrm{~A}-\mathrm{A}_{0}\right)\left(f-f_{0}\right) .
$$

Параметр $\Sigma$ в формуле (2) является неким комплексным параметром, характеризующим в данной трибосистеме микромеханику разрушения поверхности в процессе воздействия на нее потока гранул обрабатывающей среды. На основе кинетических представлений процесса разрушения поверхности установлена зависимость (5), раскрывающая физический смысл комплексного параметра $^{\Sigma}$, из которой следует, что он зависит только от свойств обрабатываемого материала и обрабатывающей среды [2]:

$$
\Sigma=H V \cdot \exp \left(\frac{\Delta H_{T S}-k_{\mathrm{T}} \cdot V_{m} H V_{\mathrm{cp}}}{R T}\right),
$$

В таблице 1 представлены величины модуля сопротивления разрушению обрабатываемых 


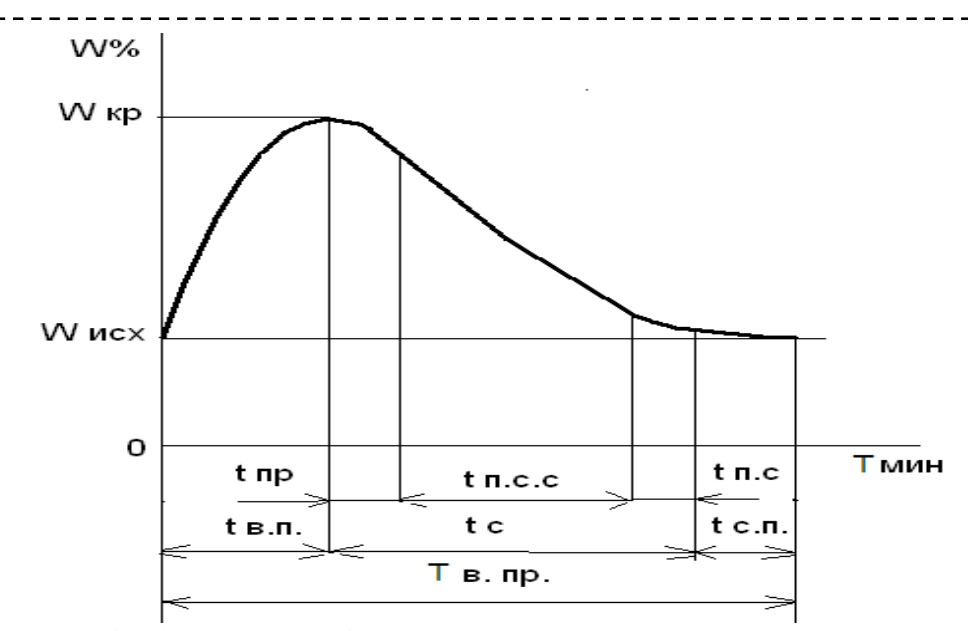

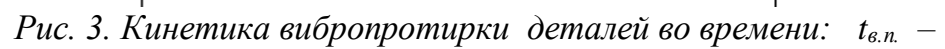
время влагопоглащения;

$T_{c}$ - время сушки детали; $t_{c . n .}-$ время сухой протирки; $t_{n p}-$ время прогрева массы загрузки; $t_{\text {n.c.c. }}$ время постоянной скорости просушки среды; $t_{n . c}-$ время падающей скорости просушки среды;; $T_{\text {в.nр. }}$ - продолжительность вибропротирки. Fig. 3. Kinetics of vibrating wiping of parts in time: tv.p. - time of moisture absorption;

Tc-part drying time; ts.p. - dry wipe time; tnp - heating time of the load mass; t p.s. - time of constant drying rate of the medium; tp $s$-time of the decreasing rate of drying of the medium; TV ave. - the duration of the vibrating wiper.

материалов при виброобработке косточковыми органическими средами, рассчитанные согласно (5).

В обобщенном виде модель, описывающая технологический эффект виброобработки косточковыми органическими средами, имеет вид:

$$
\gamma_{\mathrm{M}}=\frac{K_{\text {д }} \pi g \rho_{\mathrm{oc}} L\left(\mathrm{~A}-\mathrm{A}_{0}\right)\left(f-f_{0}\right)}{\Sigma} \mathrm{M} / \mathrm{c} ;
$$

Экспериментальная проверка аналитической модели (6), предложенной для расчета величины удельного металлосъема с поверхности образцов в процессе виброобработки косточковыми органическими средами, показала ее приемлемость для практического применения с целью оценки производительности процесса, а также определения геометрических параметров качества поверхности, формируемой в процессе виброобработки. Как следует из таблицы 2 и Рис. 2 , степень расхождения экспериментальных и расчетных значений величины скорости металлосъема находится в пределах экспериментальной ошибки [3].

\footnotetext{
Особенности вибропротирки деталей средами органического происхождения

Технологически вибропротирка деталей средами органического происхождения в виде гранул измельченных стержней початков кукурузы включает в себя два основных этапа: влагопоглощение гранулами среды жидкости с поверхности детали и непосредственно сухая протирка с целью придания детали товарного вида.
}

\section{$13-15 \%$}

Последовательность процесса вибропротирки графически можно проиллюстрировать следующим образом; выделив в нем три явно выраженных этапа (Рис. 3): влагопоглощение, при котором происходит повышение влажности органической среды до критической; просушка органической среды и снижение ее влажности до нормативной; сухая протирка [4].

Важным показателем интенсивности процесса сушки детали является удельный влагосъем жидкости с ее поверхности. По мере насыщения гранулами органической среды влагой, процесс приобретает характер влажной протирки поверхностей деталей. $\mathrm{C}$ целью исключения этого явления и осуществления сухой протирки деталей необходимо производить высушивание гранул рабочей среды таким образом, чтобы степень влажности обрабатывающей органической среды находилась на уровне предопределенных техническими условиями - не более

Для процесса просушки среды по времени характерна следующая последовательность: прогрев массы загрузки, период постоянной скорости и период падающей скорости просушки, обеспечивающий сухую протирку деталей.

При проведении вибропротирки возможны два технологических подхода к ее реализации. При первом подходе детали после виброабразивной обработки с СОЖ загружают в виброкамеру с органической средой, имеющей нормативную влажность и соответствующую окружающей среде температуру. По истечении определенного промежутка времени $\mathrm{t}_{\text {в.п }}$, устанавливаемого регламентом, включаются нагревательные элементы и происходит процесс просушки массы загрузки и последующая сухая протирка деталей. При таком подходе идет разделение во времени этапа влагонасыщения среды и сухой протирки, что влечет увеличение продолжительности процесса и, как следствие, делает его технологически не производительным.

При втором подходе влагосъем с поверхности органической средой осуществляется сразу после загрузки деталей в камеру с включенными нагревательными элементами, обеспечивающую нагрев обрабатывающей среды. Это позволяет совместить время прогрева массы загрузки с временем влагонасыщения обрабатывающей среды, не доводя ее влажность до критической, и тем самым сократить скорость периода просушки 
среды, протекающий с постоянной скоростью, при этом сократить продолжительность и повысить производительность вибропротирки. Кроме того, проведение вибропротирки с постоянно действующими нагревательными элементами, встроенными в рабочую камеру, способствует более интенсивному перемешиванию массы загрузки в связи с более ослабленными силами поверхностного взаимодействия частиц органической среды как с деталью, так и между собой.

Продолжительность цикла вибропротирки для наиболее технологичного процесса можно записать в виде:

$\mathrm{T}_{\text {в.пр. }}=\mathrm{t}_{\text {с. }}+\mathrm{t}_{\text {с.п. }}$.

Время для сухой протирки деталей должно устанавливаться экспериментально, исходя из технологических соображений, связанных с обеспечением требуемого качества поверхности конкретных деталей, и составлять от общей продолжительности определенную долю:

$$
\mathrm{t}_{\mathrm{c} . \Pi} \approx_{\mathrm{k}} \times \mathrm{T}_{\text {в.пр }}
$$

где $\mathrm{k}$ - коэффициент, устанавливающий долю в \% продолжительности сухой протирки в общей продолжительности процесса вибропротирки.

Время просушки органической среды, как показано на Рис. 3, равно:

$$
\mathrm{t}_{\mathrm{c}}=\mathrm{t}_{\text {пр }}+\mathrm{t}_{\text {п.с.с. }}+\mathrm{t}_{\text {п.с. }} \text {. }
$$

Для оценки времени прогрева массы загрузки при совпадении его с периодом влагопоглощения можно воспользоваться зависимостью:

$$
\mathrm{t}_{\text {пр }} \approx_{\mathrm{t}_{\text {вп }}=\mathrm{F}} \cdot \frac{\rho \cdot h}{q *} \mathrm{~K}_{\text {т }},
$$

где $\mathrm{F}$ - площадь поверхности обрабатываемой детали; $\rho$ - плотность жидкости; h - толщина жидкостной пленки на поверхности обрабатываемой детали; $q^{*}$ - удельное объемное поглощение жидкости с поверхности детали; К - коэффициент, учитывающий температуру подогрева обрабатывающей среды.

Для расчета продолжительности просушки рабочей среды предлагается формула в виде:

$$
\mathrm{t}_{\text {п.с.с }}=\frac{W_{\Pi \mathrm{P}}-W_{\text {Ту }}}{K_{\mathrm{X}} \cdot N},
$$

где $W_{\text {ту }}$ - влажность обрабатывающей среды, соответствующая техническим условиям, $\% ; W_{\text {ПР }}-$ предельная \% влажность обрабатывающей среды, достигаемая в процессе вибропротирки, \%; $N$ скорость сушки; $K_{\mathrm{X}}-$ коэффициент, учитывающий химико-биологические свойства обрабатывающей среды.

Рассмотрение технологических аспектов виброотделки деталей средами органического происхождения позволило сделать следующие выводы:

1. Органические среды из природных материалов вполне обоснованно могут быть применены в качестве технологических обрабатывающих сред на операциях финишной отделки деталей в условиях виброобработки.

2. Технологический эффект при обработке органическими средами обеспечивается в результате воздействия на поверхность детали стационарного потока гранулированной среды, основными характеристиками которого являются гидростатическая плотность и скорость перемещения.

3. Вибропротирка деталей органическими средами в виде дробленых гранул стержней кукурузы может выступать в качестве альтернативного способа сушки небольших по массе и габаритам маложестких деталей, позволяющей на базе виброустановок торового типа осуществлять удаление влажности с поверхности деталей, прошедших виброабразивную отделочную обработку с применением СОЖ, и может реализовываться в комплексе технологического оборудования для отделочной обработки деталей, являясь ее завершающим циклом.

\section{СПИСОК ЛИТЕРАТУРЫ}

1. Лебедев В.А. Повышение качества виброотделки поверхностей высокоточных деталей средами органического происхождения / В.А. Лебедев, Шишкина А.П., Крупеня Е.Ю. // Вестник РГАТУ им. П.А. Соловьева. - №2(41) - Рыбинск: РГАТУ, 2017. - С.132-137.

2. Шишкина А.П. Перспективы применения гранулированных сред органического происхождения в технологии виброобработки деталей / А.П. Шишкина, В.А. Лебедев, Е.Ю. Крупеня // Процессы абразивной обработки, абразивные инструменты и материалы. Шлифабразив-2014: сборник статей Международной научно-технической конференции г. Волжский, 9-11 сентября 2014 г. - Волгоград: ВолгГАСУ, 2014. - С. 131-133.

3. Шишкина А.П. Энергетические аспекты виброобработки деталей косточковыми органическими средами / А.П. Шишкина, В.А. Лебедев, М.М. Чаава // Вестник БГТУ. - №5 (58) - Брянск: БГТУ, 2017. C.42-49. 
4. Крупеня Е.Ю. Технологические аспекты вибрационной протирки деталей рабочими средами органического происхождения / Е.Ю. Крупеня, В.А. Лебедев // Известия Орел, ГТУ. - 2009. - №2 - 3/ 274 (560).

\section{REFERENCES}

1. Lebedev V.A. Improving the quality of vibro-finishing of surfaces of high-precision parts with organic media. Lebedev, A.P. Shishkina, E.Yu. Krupenya // Bulletin of RGATU im. P.A. Solovyov. - No. 2 (41) Rybinsk: RGATU, 2017. - P.132-137.

2. Shishkina A.P. Prospects for the use of organic granular media in the technology of vibration processing of parts / A.P. Shishkin, V.A. Lebedev, E.Yu. Krupenya // Processes of abrasive processing, abrasive tools and materials. Grinding abrasive-2014: collection of articles of the International Scientific and Technical Conference Volzhsky, September 9-11, 2014 - Volgograd: VolgGASU, 2014. - pp. 131-133.

3. Shishkina A.P. Energy aspects of vibration processing of parts with bone organic media / A.P. Shishkin, V.A. Lebedev, M.M. Chaava // Bulletin of BSTU. - No. 5 (58) - Bryansk: BSTU, 2017. - P.42-49.

4. Krupenya E.Yu. Technological aspects of vibration wiping of parts with working media of organic origin / E.Yu. Krupenya, V.A. Lebedev // Izvestia Orel, GTU. - 2009. - No. 2 - 3/274 (560). 